\title{
M31 gamma-ray emission - a closer look at different explanations
}

\section{Christopher Eckner*}

University of Nova Gorica, Slovenia

E-mail: christopher.eckneraung.si

\section{on behalf of the Fermi-LAT collaboration and external authors P. Serpico ${ }^{a}$,}

\section{J. Petrovic ${ }^{b}$ and T. Prodanovic ${ }^{b}$}

${ }^{a}$ Laboratoire de Physique Théorique d'Annecy-le-Vieux (LAPTh), Univ. de Savoie,

CNRS, B. P. 110, Annecy-le-Vieux F-74941, France

${ }^{b}$ Department of Physics, Faculty of Science, University of Novi Sad,

Trg Dositeja Obradovića 4, 21000 Novi Sad, Serbia

E-mail: serpico@lapth.cnrs.fr, jovana.petrovic@df.uns.ac.rs,

prodanvc@df.uns.ac.rs

\begin{abstract}
A new measurement of a spatially extended gamma-ray signal from the center of M31 was published recently (Ackermann et al. 2017, ApJ 836, 208), reporting that the emission broadly resembles the Galactic center excess (GCE) of the Milky Way (MW). In this contribution we discuss the possibilities that the signal originates from a population of millisecond pulsars (MSPs), or alternatively the annihilation of dark matter (DM) particles. As an astrophysical interpretation in terms of MSPs appears viable, we derive upper limits on the annihilation cross section in the $b \bar{b} / \tau^{+} \tau^{-}$-channel of weakly interacting massive particles (WIMPs) in a mass range from $1 \mathrm{GeV}$ to $10 \mathrm{TeV}$, taking into consideration different DM density profiles and including the effect of substructure and adiabatic contraction.
\end{abstract}

The European Physical Society Conference on High Energy Physics

5-12 July

Venice, Italy

*Speaker. 


\section{Introduction}

For nearly ten years, the Large Area Telescope mounted on the Fermi Gamma-Ray Space Telescope (Fermi-LAT) has been taking enormous amounts of high-quality data in the energy range between $20 \mathrm{MeV}$ and $300 \mathrm{GeV}$. Extensive analyses of this data revealed a gamma-ray excess around the MW's Galactic center with respect to galactic diffuse emission models and point sources. The excess' properties have been studied and established over the time, see e.g [1]. The excess was found to peak around $2 \mathrm{GeV}$ and its spatial extension is well-fit by a contracted Navarro-Frenk White DM profile. However, recent analyses claim that the GCE signal is more likely to originate from a population of unresolved point-sources within the MW's central region favoring an alternative explanation of the GCE in terms of the gamma-ray emission of MSPs [2]. Despite the controversial discussion whether or not MSPs are able to reproduce the excess' spectral and radial distribution, they are about to become the current consensus model explaining the GCE.

On the other side, a recently published analysis of Fermi-LAT [3], evaluating data taken within 88 months, was able to detect M31 in gamma-rays as a spatially extended object at $4 \sigma$ improving an old result that only yielded a marginal spatial extension. The extension is best-fit by a uniform disk of radius $0.38^{\circ} \pm 0.05^{\circ}$ corresponding to a $(5.21 \pm 0.69) \mathrm{kpc}$ region given the distance to M31 of $785 \mathrm{kpc}$. Moreover, the emission does not correlate with dust or star-forming activity in the disk. Having at hand a MW-like object whose disk does not interfere much with the emission in the center and where the region of gamma-ray emission is comparable to the size of the MW bulge, we can cross-check if a certain model that works for one of the galaxies is also consistent with the measurement of the other one. To this end, we want to explore in more detail two possible explanations that could account for the GCE as well as the extended emission of M31: MSPs inside the bulges of both galaxies and DM annihilation in the central regions of M31.

\section{Millisecond pulsar emission in the Milky Way and M31}

To investigate the pulsar explanation in both galaxies, we consider the formation of MSPs inside the bulge of the respective galaxy only. ${ }^{1}$ In general, there are two ways in which an MSP can emerge from a former pulsar: (i) The progenitor of the pulsar was already part of a tightly bound binary system with an extreme mass ratio which stayed intact even after the supernova. This system would then naturally evolve into an MSP. The number of MSPs due to this primordial formation would be proportional to the stellar mass in the region of interest; (ii) due to a two-body encounter of a pulsar with another star inside the galactic bulge a binary system is formed. The probability of such a formation scales with the square of the stellar density $\rho_{*}$ in the region of interest but is suppressed by the mean velocity dispersion $\sigma$ of stars in that area because it weakens the gravitational focusing. This channel was assigned the name dynamical formation and there are experimental confirmations of this process [5].

Our goal is to estimate the luminosity in gamma-rays of the galactic bulges of the MW and M31. The stellar density in this part of a galaxy is usually higher than elsewhere so that it is quite likely that on top of primordially formed MSPs we can also expect a non-negligible number of

\footnotetext{
${ }^{1}$ There might be the possibility of deposition of MSPs due to infalls of globular clusters which get disrupted by tidal forces [4].
} 
dynamically formed MSPs.

In order to quantify the impact of both formation mechanisms we suggest a simple toy model for the overall number density of MSPs $n_{\text {MSP }}$ inside the bulges which is given by

$$
n_{\mathrm{MSP}}(r)=A\left\langle\rho_{*}(r)\right\rangle_{d_{1}}+B\left\langle\rho_{*}(r)^{2}\right\rangle_{d_{2}} / \sigma
$$

where $r$ denotes the distance to the Galactic center, $A$ and $B$ are normalization constants and $\langle\cdot\rangle_{d}$ indicates that we are applying a smoothing to the stellar densities because MSPs obtain a "kickvelocity" in the moment of their birth so that they migrate from their original birthplace depending on the magnitude of the velocity. For the properties of the stellar density in the MW bulge and its nuclear stellar cluster (NSC), a region of the enhanced stellar density found in the inner $\sim 10 \mathrm{pc}$, we use the reported values in [6] whereas in the case of M31 we adopt the results of [7]. By taking into account kick velocity estimates reported in the literature, we estimate that $d_{1}=700-900 \mathrm{pc}$, while $d_{2}$ is negligible.

PRIMORDIALLY FORMED MSPS: We use the local information about 66 MSPs in the MW disk which yields an approximation of the gamma-ray luminosity function $\left(\mathrm{d} N / \mathrm{d} L_{\gamma}\right)_{\mathrm{MW}}$ of the MW. Following the approach of [8], we are able to predict the MSP luminosity of the MW bulge $\left\langle L_{\mathrm{b}}^{\mathrm{prim}}\right\rangle$ by a rescaling to the mass of the bulge using

$$
\left\langle L_{\mathrm{b}}^{\mathrm{prim}}\right\rangle=\frac{M_{\mathrm{MW}, \mathrm{b}}}{M_{\mathrm{MW}}} \int_{L_{\min }}^{L_{\max }} L_{\gamma}\left(\frac{d N}{d L_{\gamma}}\right)_{\mathrm{MW}} d L_{\gamma},
$$

where $M_{\mathrm{MW}}$ and $M_{\mathrm{MW}, \mathrm{b}}$ are the total mass of the MW and the bulge mass of the MW, respectively. The idea is to recycle this method also in the case of M31 where we simply rescale the expression to $M_{\mathrm{M} 31, \mathrm{~b}}$ instead of $M_{\mathrm{MW}, \mathrm{b}}$. Our strategy is based on the fact that the primordial channel is proportional to the stellar density of the bulge or equivalently the mass inside this object. In this way, we obtain the luminosity that can be expected from the "A-term" of Eq. 2.1.

Taking into account the uncertainties associated with the used quantities, we derived for our own galaxy $L_{\mathrm{MW} \text { bulge }}^{\text {prim }}=2.0_{-1.3}^{+3.2} \times 10^{37} \mathrm{erg} \mathrm{s}^{-1}$ and for M31 on the other side, $L_{\mathrm{M} 31 \text { bulge }}^{\text {prim }}=$ $7.5_{-5.3}^{+12.0} \times 10^{37} \mathrm{erg} \mathrm{s}^{-1}$. The interpretations of both estimates are different in the following sense: The available experimental measurement of the MSP-like gamma-ray luminosity in a region $20^{\circ}$ around the MW's Galactic center is about $L_{\mathrm{MW}, \lesssim 20^{\circ}}^{\mathrm{obs}}=2.2_{-1.9}^{+1.5} \times 10^{37} \mathrm{erg} \mathrm{s}^{-1}$ [1]. Thus, the primordial channel can fully explain this emission within the derived uncertainty. However, under the assumption that the total observed emission from M31 is only due to an MSP-like component, we find the luminosity of the signal to be much higher than $L_{\mathrm{M} 31 \text { bulge, namely } L_{\mathrm{M} 31}^{\mathrm{p}} \text { bulge }}^{\text {prim }}=$ $(28 \pm 4) \times 10^{37} \mathrm{erg} \mathrm{s}^{-1}$. Although our prediction of the luminosity of M31's bulge exhibits large uncertainties, we are only consistent with the observation at a $2 \sigma$ level. This result is not completely unexpected since there are other sources of gamma-rays that could add to the signal. Also, the contribution from the dynamical channel could make the discrepancy less significant.

DYNAMICALLY FORMED MSPS: The most dominant contribution to dynamical formation comes from the NSC. As densities of NSCs are comparable to those of globular clusters, where dynamical formation dominates, we use the correlation between the stellar density and gamma-ray luminosity observed in globular clusters to estimate the gamma-ray luminosity (due to dynamical formation) of these environments. This correlation was studied in [5] and we display in Fig. 1 (left) 
its application to the NSCs of both galaxies. The result of this approximation indicates that the luminosity from the dynamical channel is only about $5 \%$ of the primordial channel's luminosity for each galaxy under study. Hence, we do not spoil our finding for the MW but, on the other hand, our prediction for M31 is still consistent with the observation only at a $2 \sigma$ level so that there is space for additional contributions apart from MSPs.

In Fig. 1 (right) we show the radial profile of the primordial and dynamical formation channel (where the extent of the NSC is dominated by the LAT PSF), normalizing the "A-term" to reproduce the overall luminosity calculated previously. We find that the predictions fit the observed emission quite well and they do not overshoot the upper limit on the gamma-ray flux of the Galactic center within the innermost $2^{\circ}$ displayed as a red point.

Although there is no analog of the radial profile of the GCE for M31, we can make use of the morphology analysis of the M31's gamma-ray emission in [3]. Besides the uniform disk template which yields the best-fit result to the LAT measurement, the authors studied other templates like the Spitzer/IRAC maps at $3.6 \mu \mathrm{m}$ which trace old stars that dominantly reside inside M31's bulge. In fact, this template provides a better fit to the gamma-ray signal than maps with star formation tracers and gas in M31.

Our model predicts an emission of M31 due to a population of unresolved point sources which follows the stellar density in the bulge and which needs to be smoothed with a kpc-kernel due to the migration of primordial MSPs. Hence, the accordingly smoothed Spitzer/IRAC maps at 3.6 $\mu \mathrm{m}$ provide a template capturing the basic prediction of our toy model. Repeating the morphology analysis of [3] with this template improves the fit of the unsmoothed IRAC maps but the resulting test statistics is within $2.4 \sigma$ of the best-fit test statistics of the uniform disk template.
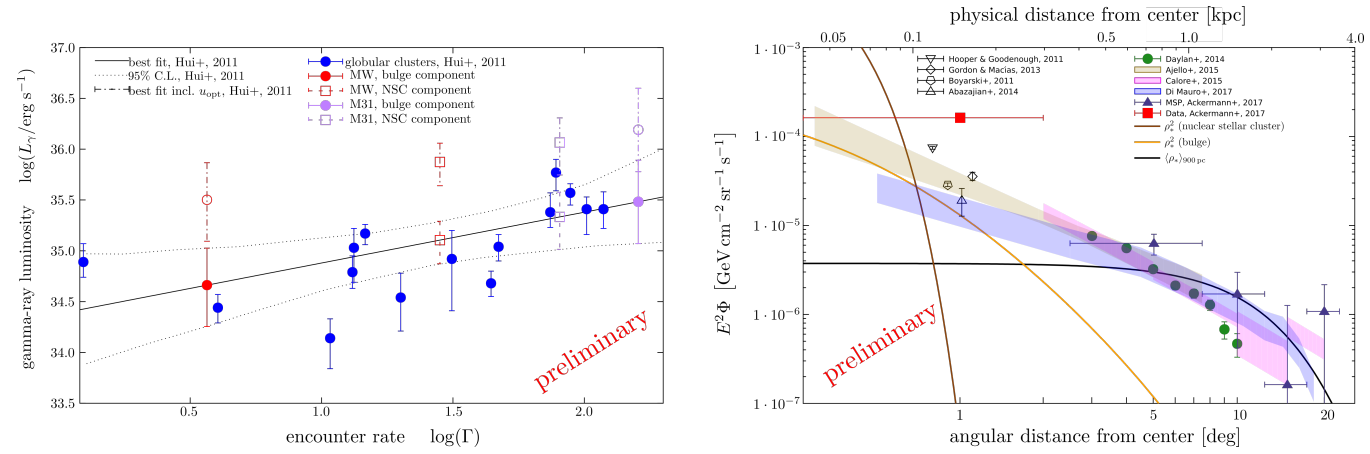

Figure 1: (Left) Scaling relation of gamma-ray luminosity $L_{\gamma}$ of globular clusters with the stellar encounter rate, from [5]. The points for inner bulge regions and NSCs of MW (red circles and squares) and M31 (purple circles and squares) are added according to the chosen stellar density profiles. (Right) Radial profile of the GCE emission, shown together with the best fit model from Eq. 2.1 using a smoothing scale $d_{1}=900 \mathrm{pc}$ (black line) and $d_{2}=0 \mathrm{pc}$ (orange $\&$ brown lines). The normalization of the $\rho_{*}$-term is chosen to match the data of Calore 2015 ([9]), while the normalization of the $\rho_{*}^{2}$-terms is derived relative to the findings of the left plot.

\section{M31 gamma-ray emission from DM}

An explanation of the GCE by DM annihilation cannot be excluded from the current data, thus 
it is an intriguing question if the observed gamma-ray emission of M31 is also consistent with the state-of-the-art limits on the thermally-averaged annihilation cross section of (WIMP) DM. In fact, according to the DM template fit of [3], this scenario cannot be excluded and a more detailed study is necessary.

The first step in our study concerns the modeling of the theoretically expected gamma-ray flux from M31. This flux takes into account the particle physics properties of DM and its astrophysical distribution in the DM halo:

$$
\frac{\mathrm{d} \Phi}{\mathrm{d} E_{\gamma}}=\frac{\langle\sigma v\rangle}{8 \pi m_{\chi}^{2}} \sum_{i} \mathrm{R}_{i} \frac{\mathrm{d} N_{i}}{\mathrm{~d} E_{\gamma}} \times\left(\int_{\Delta \Omega} \int_{1.0 . \mathrm{s} .} \rho_{\chi}^{2}(\vec{l}) \mathrm{d} l \mathrm{~d} \Omega\right),
$$

where $m_{\chi}$ denotes the mass of the DM particle, $\langle\sigma v\rangle$ the annihilation cross section, $\mathrm{R}_{i}$ the branching ratio to the $i^{\text {th }}$ annihilation channel and $\mathrm{d} N_{i} / \mathrm{d} E_{\gamma}$ its differential yield of gamma-rays from a single DM annihilation event which we take from PPPC4DMID [10]. $\rho_{\chi}(\vec{l})$ refers to the DM density distribution of the object under study. This density distribution is part of the term in parenthesis which is usually dubbed $J$-factor. As the $J$-factor depends on the squared DM density profile it is very sensitive to overdensities along the line of sight. M31 is centered inside a large DM halo but such a large parent halo hosts a large number of small subhalos and even further levels of substructure. Those subhalos will lead to a substantial boost of the host halo's $J$-factor. Thus, it will be important to carefully model the substructure according to recent results of $N$-body simulations as well as astronomical observations.

To account for the uncertainty of the DM distribution in M31, we define two limiting DM templates (MAX and MIN) which include both a smooth component and a substructure component. Moreover, we also create a benchmark template (MED) that states a realistic DM distribution in M31 based on recent observations and simulations. We will use the publicly available code CLUMPY [11] to generate $J$-factor maps of M31.

As a matter of fact, M31 seems to be the only well-studied galaxy which was argued to require the effect of adiabatic contraction around its central region [12]. Therefore, we adopt this profile as the smooth component in our benchmark (MED) template. The smooth DM component of the remaining two models are taken from [7]. We find that the Burkert profile minimizes and the Einasto profile maximized the total emission among the listed DM distributions. These profiles are totally specified by their scale radius $r_{s}$ and density normalization $\rho_{0}$ given in Tab. 1 .

We need to specify several parameters that govern the amount and distribution of subhalos in a given object. The parameters with the largest impact are:

- The index $\alpha$ of the subhalo mass function $\mathrm{d} n / \mathrm{d} M$ following a power-law,

- the fraction of the DM halo mass which is stored in substructure $f_{\text {sub }}$,

- the minimal mass of DM subhalos $M_{\min }$ and

- the subhalo concentration parameter $c_{200}$.

We rely on the most recent model of the concentration parameter of subhalos ${ }^{2}$ [13] and we show in Tab. 1 our definitions of the three models which are based on Fig. 7 in [14] to account for the mutual dependencies among the remaining three parameters.

\footnotetext{
${ }^{2}$ To this end, we make use of a developer's version of CLUMPY which already features this concentration model.
} 

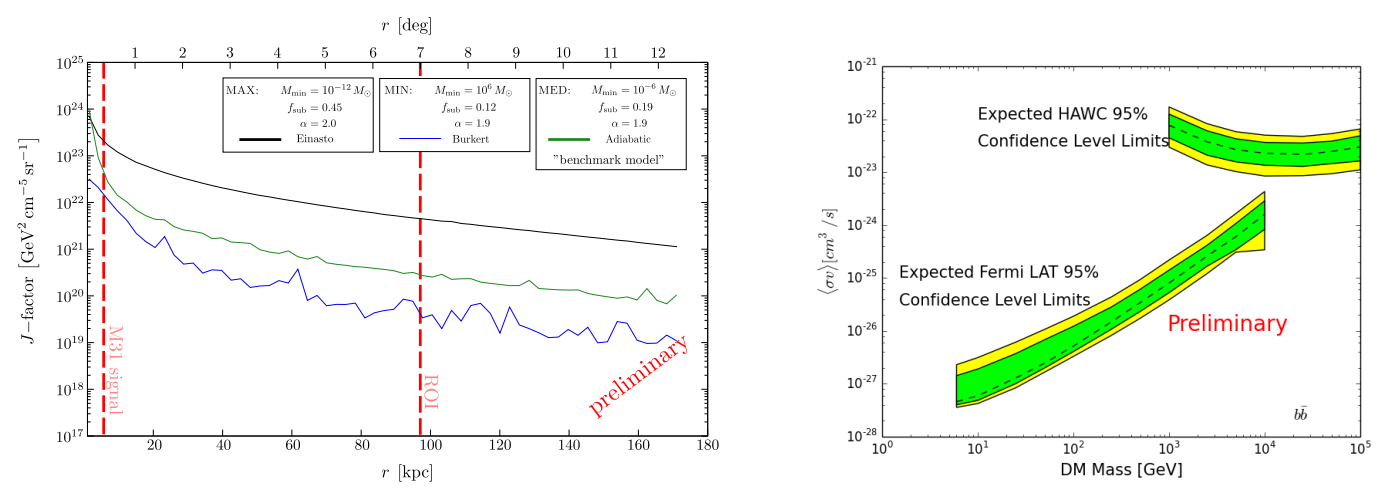

Figure 2: (Left) Radial $J$-factor profiles using the three DM templates of M31. The two vertical dashed lines in red display the spatial extension of the gamma-ray signal from M31 and our ROI of $14^{\circ} \times 14^{\circ}$ up to which we perform the analysis, respectively. (Right) Expected Fermi-LAT and HAWC sensitivities for DM annihilating into $b \bar{b}$ and assuming a DM distribution in M31 according to the MED model. The yellow bands display the $68 \%$ C.L. whereas the green bands show the $95 \%$ C.L.

\begin{tabular}{|c|c|c|c|c|c|}
\hline & smooth profile & $\alpha$ & $f_{\text {sub }}$ & $M_{\min }\left[M_{\odot}\right]$ & $c_{200}$ \\
\hline \hline MIN & $\begin{array}{c}\text { Burkert } \\
r_{s}=9.06 \mathrm{kpc}, \rho_{0}=3.68 \times 10^{7} M_{\odot} / \mathrm{kpc}^{3}\end{array}$ & 1.9 & 0.12 & $10^{6}$ & \\
\cline { 1 - 5 } MAX & $\begin{array}{c}\text { Einasto } \\
r_{s}=178 \mathrm{kpc}, \rho_{0}=8.12 \times 10^{3} M_{\odot} / \mathrm{kpc}^{3}\end{array}$ & 2.0 & 0.45 & $10^{-12}$ & \multirow{2}{*}{ [13] } \\
\cline { 1 - 4 } MED & $\begin{array}{c}\text { adiabatically contracted NFW } \\
r_{s}=25.65 \mathrm{kpc}, \rho_{0}=4.44 \times 10^{7} M_{\odot} / \mathrm{kpc}^{3}\end{array}$ & 1.9 & 0.19 & $10^{-6}$ & \\
\hline
\end{tabular}

Table 1: Summary of the three DM models for M31.

The LAT analysis closely follows the approach of [15] which was used to derive bounds on the DM annihilation cross section analyzing the gamma-ray emission from the Small and Large Magellanic Clouds. We scan the parameter space of annihilating DM with masses from $10 \mathrm{GeV}$ to $10 \mathrm{TeV}$ while focusing on the $b \bar{b}$ and $\tau^{+} \tau^{-}$channels. An estimate of the sensitivity of the MED template to DM annihilations into $b \bar{b}$ is shown in Fig. 2 together with the estimate of the HAWC observatory, which will provide complementary limits at higher masses.

\section{Acknowledgements}

The Fermi-LAT Collaboration acknowledges support for LAT development, operation and data analysis from NASA and DOE (United States), CEA/Irfu and IN2P3/CNRS (France), ASI and INFN (Italy), MEXT, KEK, and JAXA (Japan), and the K.A. Wallenberg Foundation, the Swedish Research Council and the National Space Board (Sweden). Science analysis support in the operations phase from INAF (Italy) and CNES (France) is also gratefully acknowledged. This work performed in part under DOE Contract DE-AC02-76SF00515. 


\section{References}

[1] FERMI-LAT collaboration, The Fermi Galactic Center GeV Excess and Implications for Dark Matter, Astrophys. J. 840 (2017) 43, [1704.03910].

[2] FERMI-LAT collaboration, Characterizing the population of pulsars in the Galactic bulge with the Fermi Large Area Telescope, Submitted to: Astrophys. J. (2017) , [1705. 0000 9].

[3] FERMI-LAT collaboration, Observations of M31 and M33 with the Fermi Large Area Telescope: A Galactic Center Excess in Andromeda?, Astrophys. J. 836 (2017) 208, [1702. 08602 ].

[4] T. D. Brandt and B. Kocsis, Disrupted Globular Clusters Can Explain the Galactic Center Gamma Ray Excess, Astrophys. J. 812 (2015) 15, [1507.05616].

[5] C. Y. Hui, K. S. Cheng, Y. Wang, P. H. T. Tam, A. K. H. Kong, D. O. Chernyshov et al., The Fundamental Plane of Gamma-ray Globular Clusters, Astrophys. J. 726 (Jan., 2011) 100, [1101.4107].

[6] E. Vanhollebeke, M. A. T. Groenewegen and L. Girardi, Stellar populations in the Galactic bulge, ArXiv e-prints (Mar., 2009), [0903.0946].

[7] A. Tamm, E. Tempel, P. Tenjes, O. Tihhonova and T. Tuvikene, Stellar mass map and dark matter distribution in M31, Astron. Astrophys. 546 (2012) A4, [1208. 5712].

[8] M. Winter, G. Zaharijas, K. Bechtol and J. Vandenbroucke, Estimating the GeV Emission of Millisecond Pulsars in Dwarf Spheroidal Galaxies, Astrophys. J. 832 (2016) L6, [1607 . 06390 ].

[9] F. Calore, I. Cholis and C. Weniger, Background Model Systematics for the Fermi GeV Excess, JCAP 1503 (2015) 038, [1409.0042].

[10] M. Cirelli, G. Corcella, A. Hektor, G. Hutsi, M. Kadastik, P. Panci et al., PPPC 4 DM ID: A Poor Particle Physicist Cookbook for Dark Matter Indirect Detection, JCAP 1103 (2011) 051, [1012.4515].

[11] V. Bonnivard, M. Hütten, E. Nezri, A. Charbonnier, C. Combet and D. Maurin, CLUMPY : Jeans analysis, $\gamma$-ray and $v$ fluxes from dark matter (sub-)structures, Comput. Phys. Commun. 200 (2016) 336-349, [1506.07628].

[12] O. Y. Gnedin, D. Ceverino, N. Y. Gnedin, A. A. Klypin, A. V. Kravtsov, R. Levine et al., Halo Contraction Effect in Hydrodynamic Simulations of Galaxy Formation, 1108.5736.

[13] A. Moliné, M. A. Sánchez-Conde, S. Palomares-Ruiz and F. Prada, Characterization of subhalo structural properties and implications for dark matter annihilation signals, Mon. Not. Roy. Astron. Soc. 466 (2017) 4974-4990, [1603.04057].

[14] V. Springel, J. Wang, M. Vogelsberger, A. Ludlow, A. Jenkins, A. Helmi et al., The Aquarius Project: the subhalos of galactic halos, Mon. Not. Roy. Astron. Soc. 391 (2008) 1685-1711, [0809. 0898 ].

[15] R. Caputo et al., Search for Gamma-ray Emission from Dark Matter Annihilation in the Small Magellanic Cloud with the Fermi Large Area Telescope, Phys. Rev. D93 (2016) 062004, [1603.00965]. 